\title{
Abbildende Wirbelstromsensoren zur hochauflösenden berührungslosen Abbildung von elektrischen Eigenschaften schlecht leitender Objekte
}

\author{
Henning Heuer ${ }^{1}$, Martin Schulze ${ }^{1}$, Marcus Klein ${ }^{2}$ \\ ${ }^{1}$ Fraunhofer Institut für zerstörungsfreie Prüfverfahren FhG IZFP Dresden, Maria-Reiche Str. 2, 01109 \\ henning.heuer@izfp-d.fraunhofer.de \\ ${ }^{2}$ Suragus GmbH, Dresden, Krügerstraße 22, 01326, http://www.suragus.de
}

\section{Einleitung}

Viele Verbundwerkstoffe (z.B. Kohlenstofffaserverbundwerkstoffe) und Schichtsysteme (z.B. Photovoltaikzellen), die eine elektrische Leitfähigkeit aufweisen, lassen sich mit Hilfe fortgeschrittener Wirbelstromprüftechnik charakterisieren. Dieser Beitrag berichtet über eine neu entwickelte, abbildende Hochfrequenz-Wirbelstrommethode, mit der insbesondere schlecht leitfähige Objekte zerstörungsfrei geprüft werden können, die mit konventioneller Wirbelstromtechnik bisher nicht prüfbar waren. Damit können die Vorteile der Wirbelstromprüftechnik wie z.B. Kontakt- und Koppelmittelfreiheit, Schnelligkeit, Automatisierbarkeit auch in der Massenfertigung von Solarzellen oder CFK Strukturen genutzt werden. Zur Messung von sehr kleinen Leitfähigkeitsänderungen oder der Dicke sehr dünner Schichten, werden Wirbelstromverfahren mit sehr hohen Messfrequenzen benötigt. Die Frequenz mit der die Wirbelströme angeregt werden, beeinflusst die Eindringtiefe $\delta$ mit $\delta$ $\sim 1 / f$ (Skineffekt) und die durch den sich zeitlich ändernden Fluss $U_{\text {ind }}=-d \Phi / d t$ die Signalamplitude in der Messspule. D.h. mit steigernder Frequenz nehmen die Eindringtiefe ab und die Signalamplitude zu. Das Fraunhofer IZFP in Dresden hat ein Gerätesystem entwickelt, mit dem die zerstörungsfreie Prüfung im Frequenzbereich bis $100 \mathrm{MHz}$ Anregungsfrequenz ermöglicht wird. Die Gerätesystemplattform „EddyCus $®$ beinhaltet angepasste Sensorik, Elektronik, Applikations- und Kalibrierungs-Prozeduren sowie Auswertealgorithmen. Zur Erstellung von Abbildern der elektrischen Eigenschaften von zu prüfenden Objekten werden ortsauflösende Wirbelstromsensoren mit einem mechanischen Manipulator über die Oberfläche des Prüfobjektes bewegt. Durch Auftragen der Messsignale über dem Messort werden C-Scans der Messwerte erzeugt. Zur Reduzierung von Scanzeit und für Inline-Anwendungen können auch Linien- oder Matrix- Sensoren verwendet werden. $[1,2,3,4]$

\section{Grundlagen der Wirbelstromprüfung}

Wirbelstromverfahren sind induktive Verfahren zur zerstörungsfreien Prüfung von elektrisch leitenden und / oder ferromagnetischen Werkstoffen. Dabei wird mittels elektromagnetischer Felder die Leitfähigkeit oder Permeabilität von Werkstoffen analysiert.

Das Anwendungsspektrum von Wirbelstromverfahren lässt sich je nach Prüfaufgaben wie folgt gliedern:

- Fehlerprüfung: Nachweis von Rissen, Poren, Einschlüssen

- Werkstoff-Charakterisierung bzw. Verwechslungsprüfung

- Bestimmung der Werkstoffparameter: elektrische Leitfähigkeit $\sigma$ und magnetische Permeabilität $\mu$

- Bestimmung weiterer Werkstoffeigenschaften, die mit der elektrischen Leitfähigkeit, der magnetischen Permeabilität bzw. ihrer örtlichen Verteilung korreliert sind (Qualitätsmerkmale)

- Mechanische Härte, Einhärtetiefe, Gefügecharakterisierung

- Geometrieprüfung, z.B. die Bestimmung der Dicke von Metallen und Folien

- Bestimmung der Dicke von Isolationsschichten

- Detektion und Ortung von Metallgegenständen

Die Abb. 1 zeigt eine vereinfachte Messanordnung zur Durchführung der Wirbelstromprüfung. Ein elektrischer Wechselstrom der Stärke I und der Frequenz f fließt in der Senderspule und erzeugt in der nahen Umgebung dieser Spule ein elektromagnetisches Wechselfeld. Dieses breitet sich im Bereich der Sendespule ebenso aus wie im Bereich der Empfangsspule sowie im Prüfobjekt und wird von der 
elektrischen Leitfähigkeit $\sigma$, der magnetischen Permeabilität $\mu$ und von der Geometrie des Prüfobjekts beeinflusst. Als Empfänger kann ebenfalls eine Induktionsspule eingesetzt werden, in der das elektromagnetische Feld eine elektrische Spannung induziert. Darüber hinaus kann als Empfänger auch ein nichtinduktiver Magnetfeldsensor verwendet werden, der die Magnetfeldstärke in eine elektrische Spannung umsetzt [5].
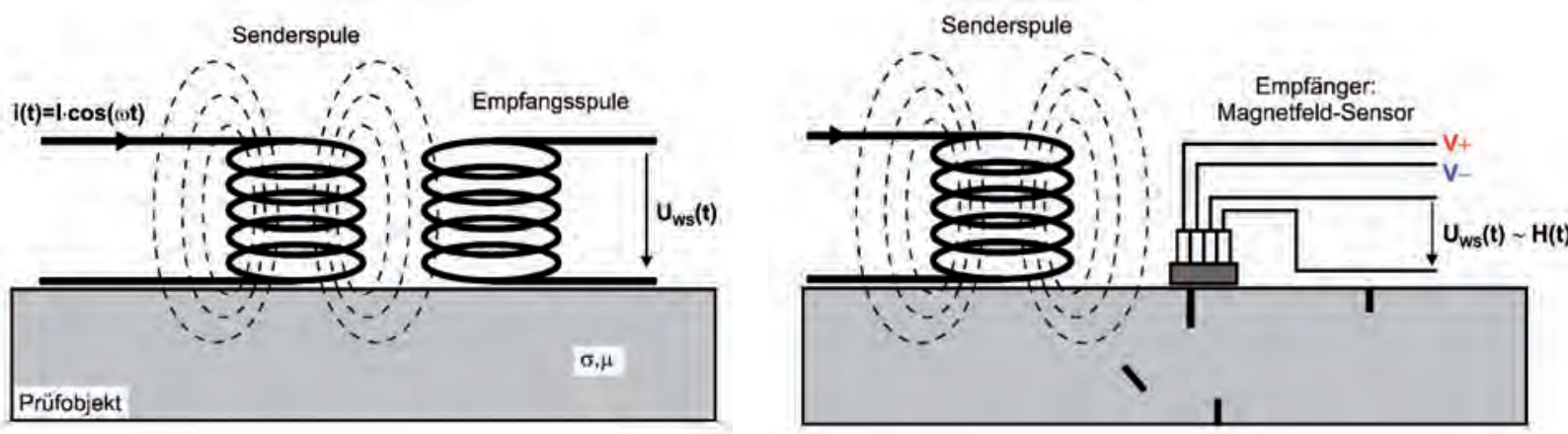

Abb. 1 Schematische Darstellung des Wirbelstromverfahrens, links mit einer Spule als Empfänger, rechts mit einem nicht-induktiven Magnetfeldsensor [4]

Ändert sich die Leitfähigkeit oder Permeabilität des Prüflings durch Materialänderungen oder z. B. durch Risse, ändert sich die Ausprägung des Feldes und damit auch die induzierte Spannung in der Empfangsspule. Diese Spannungsänderung wird aufgezeichnet. Dabei hat das elektromagnetische Feld im Prüfling eine gewisse Eindringtiefe, die nach dem sog. Skin-Effekt von der Messfrequenz, der elektrischen Leitfähigkeit und der Permeabilität abhängt. Fehler, die tiefer liegen als die Eindringtiefe, können mit dem Wirbelstromverfahren nicht detektiert werden. Abb. 2 verdeutlicht diesen Sachverhalt.

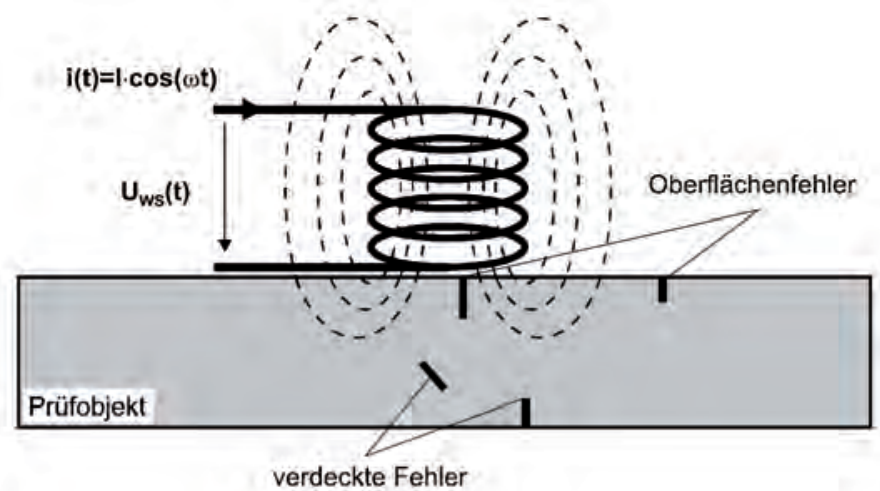

\section{Abb. 2 Schematische Darstellung des Wirbelstromverfahrens zur Rissprüfung: oberflächennahe Fehler können gut detektiert werden, Fehler unterhalb der Eindringtiefe des elektromagnetischen Feldes dagegen nicht [4]}

Als Wirbelstromsensor (WS-Sensor) wird die Anordnung eines Senderelements (Senderspule) zusammen mit einem Empfängerelement (Empfängerspule bzw. nichtinduktiver Magnetfeldsensor) bezeichnet. In der Praxis werden darüber hinaus WS-Sensoren eingesetzt, die aus mehreren Senderelementen und / oder Empfängerelementen bestehen (Differenz- und Multidifferenz-Sensoren, Kompensationsspulen, Sensorarrays etc.) sowie Ferritkerne und Abschirmungen enthalten können. Die Wirbelstromprüfung vermag wesentlich mehr zu leisten, als in den bisher dargestellten klassischen Anwendungen. Wichtige neue Anwendungsfelder sind:

- Charakterisierung von dünnen, leitfähigen Schichten auf leitfähigen Substraten Qualitätsprüfung von Kohlefaserverbundwerkstoffen

- Qualitätsprüfung von komplexen Schichtsystemen wie Solarzellen

- Schichtwiderstandsprüfung

- Tiefenprofile von Härte, Verfestigung 
Zur Messung von sehr kleinen Leitfähigkeitsänderungen, z. B. von Materialeigenschaften oder der Dicke sehr dünner Schichten, in sehr oberflächennahen Schichten auf den Bauteilen oder in Bauteilen mit sehr geringer Leitfähigkeit, werden Wirbelstromanwendungen mit sehr hohen Messfrequenzen benötigt. Das Fraunhofer IZFP in Dresden hat ein Gerätesystem mit bis zu $100 \mathrm{MHz}$ Anregungsfrequenz entwickelt, mit welchem die zerstörungsfreie Prüfung mittels Hochfrequenzwirbelstrom ermöglicht wird. Dieses Gerätesystem „EddyCus ${ }^{\circledR}$ Pro 100“ beinhaltet spezielle Sensorik, Elektronik, Applikations- und Kalibrierungs-Prozeduren sowie angepasste Auswertealgorithmen. Im Folgenden werden einige Beispiele zur Anwendung des HochfrequenzWirbelstromprüfverfahrens dargestellt.

\section{Anwendung der abbildenden Wirbelstromprüfung}

\subsection{Prinzip}

Zur Erstellung von Abbildern der elektrischen Eigenschaften von zu prüfenden Objekten werden hochauflösende Sensoren mit einem mechanischen Manipulator über die Oberfläche des Prüfobjektes bewegt. Durch Auftragen der Messsignale über dem Messort werden C-Scans der Wirbelstromparameter erzeugt. Zur Reduzierung von Scanzeit und für In-line-Anwendungen können auch Linien- oder Matrix- Sensoren verwendet werden (Abb. 3).

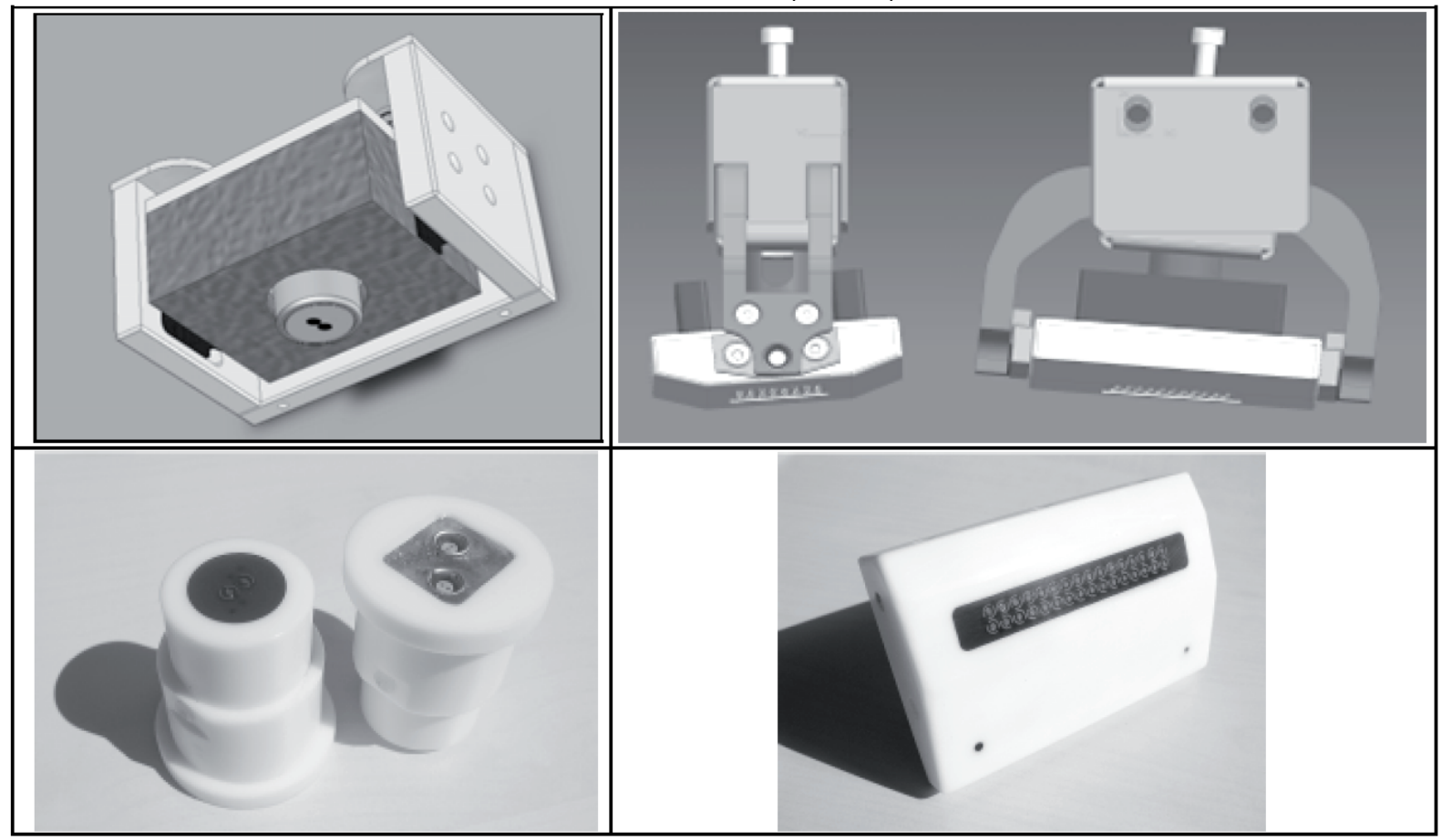

Abb. 3 Sensoren und adaptive Halterungen zur abbildenden Wirbelstromprüfung (links Einzelsensoren, rechts - Sensorarray)

\subsection{Systemvorstellung EddyCus $₫$ MPECS}

Der universelle Wirbelstrommessplatz EddyCus ${ }^{\circledR}$ MPECS (Multi Parameter Eddy Current Scanner) wurde entwickelt, um schnell und einfach Leitfähigkeitsbilder von strukturierten und unstrukturierten Schichten sowie mehrlagigen Schichtsystemen zu erstellen (Abb. 4). Da verschiedene Qualitätsparameter über die Abbildung der lokalen Leitfähigkeit bestimmt werden können, kommt das Gerät in vielen verschiedenen Industrien zur Anwendung. Das Gerät scannt mit 4 Frequenzen die zu charakterisierenden Prüfobjekte ab, wodurch zeitgleich Informationen aus unterschiedlichen Tiefen gewonnen und dargestellt werden. Die umfangreiche, aber einfach zu bedienende Software unterstützt die Separation verschiedener Leitfähigkeitseffekte und erlaubt so die gezielte Auswertung bestimmter Eigenschaften. 


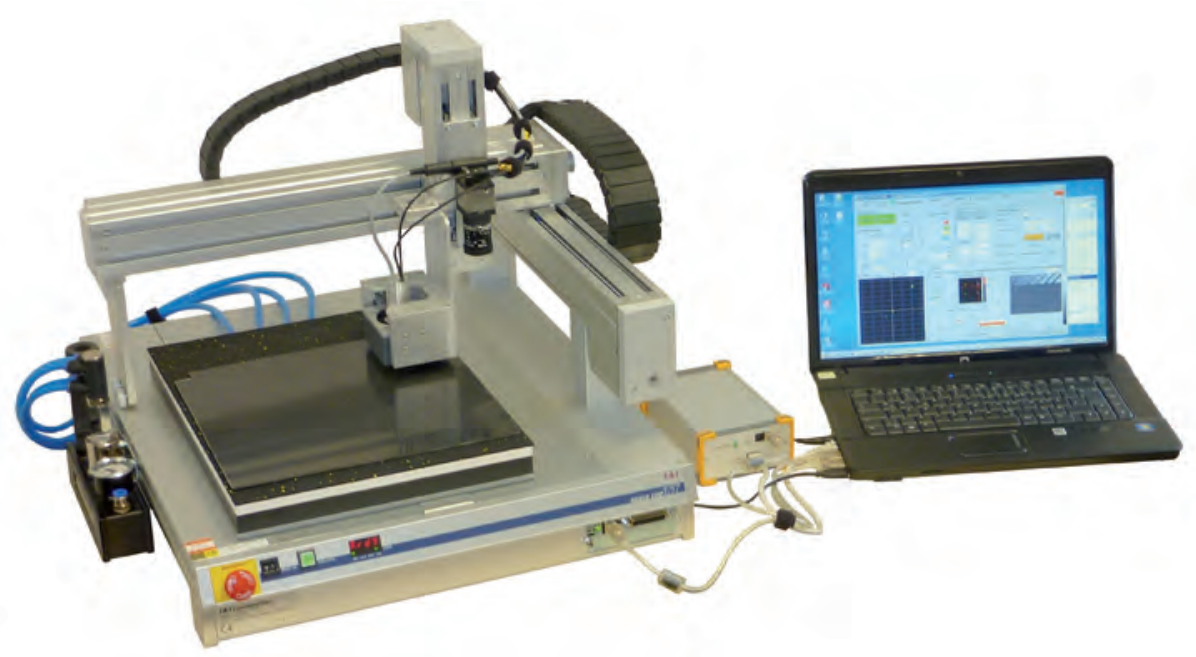

Abb. 4 EddyCus ${ }^{\circledR}$ MPECS - Abbildendes Wirbelstromprüfsystem

Dieses universelle Tischsystem vereint die Vorteile durch aktuelle Weiterentwicklungen und Trends in der Wirbelstromprüfung. Wichtige Eigenschaften sind [5]:

- Hochfrequenzmesstechnik (bis $100 \mathrm{MHz}$ )

- Genaue x-y-z Positionierung der Sensorik,

- Elegante, einfache anwenderfreundliche Parametrierung und Auswertung

- $\quad$ Automatische Bilderzeugung

- Zubehör optimiert für Anwendung (Vakuumtisch für flexible Substrate)

\section{Wirbelstromprüfung von Kohlefaserverbundwerkstoffen}

CFK Verbundwerkstoffe gewinnen, getrieben durch den Bedarf nach Leichtbauanwendungen, zunehmend an Bedeutung.Sowohl die Luftfahrt- als auch die Automobilindustrie stellen Forderungen an die Volumeninspektion von CFK Baugruppen. Hier stößt das derzeit weitverbreitete bildgebende Phased- und Sampling Phased Array Ultraschallverfahren insbesondere bei sehr dünnen Platten aufgrund der physikalisch bedingten toten Zone im Nahfeldbereich und der benötigten nassen Ankopplung an physikalische Grenzen.

Ein bedeutendes Prüfmerkmal von CFK Verbundwerkstoffen für die Gewährleistung der Stabilität und Widerstandsfähigkeit gegen Zug- und Druckkräfte ist die genaue Orientierung der gestapelten Kohlefasergelege zueinander. Die Abweichung von wenigen Graden schwächen diese strukturellen Eigenschaften bereits stark. Weitere Prüfmerkmale sind Delaminationen, Faserbrüche, Ondulationen, Impact-Schäden und die tatsächliche Dicke der CFK-Struktur. Die Abbildung 5 zeigt typische zu prüfende Fehlerbilder.
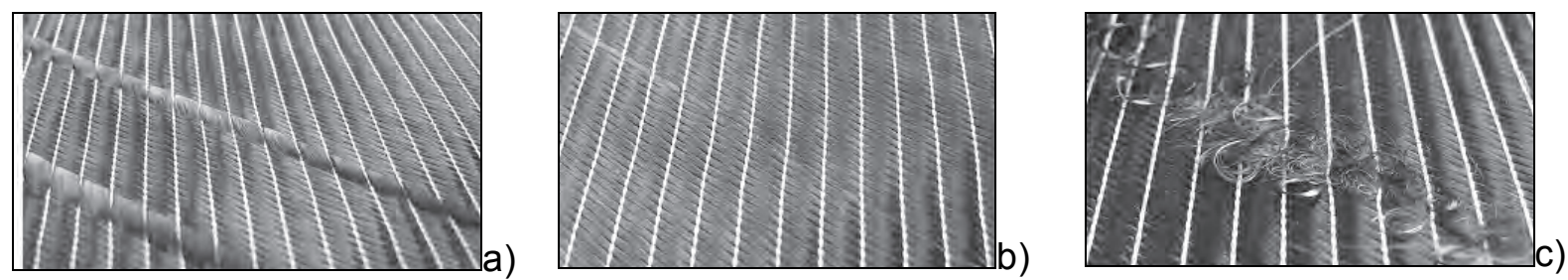

Abb. 5 Typische Defekte in Kohlefaserrohgelegen a) fehlende Faserbündel, b) Gassen, c) Ausfransungen

Kohlenstofffasern weisen eine elektrische Leitfähigkeit (Graphit $\sim 3 \times 10^{\wedge} 6 \mathrm{~S} / \mathrm{m}$ ) von ca. 1/10 der Leitfähigkeit von Aluminium auf. Werden Kohlenstofffasern in einen Kunststoff eingebettet und zu kohlenstofffaserverstärkten Kunstoffen verarbeitet, verringert sich die Summenleitfähigkeit auf ca. 1/1000 der Leitfähigkeit von Aluminium. Die Summenleitfähigkeit ergibt sich aus der Häufigkeit und 
der Leitfähigkeit der Kohlenstofffasern in einem Probenvolumen, der Übergangswiderstände benachbarter Fasern und der kapazitiven Kopplung der Fasern über den eingebrachten Kunststoff (oder Luft im Falle eines textilen Ausgangsmaterials). Wird dieses Material von außen einem elektromagnetischen Wechselfeld ausgesetzt, wird in den Kohlenstofffasern eine Spannung induziert, die zu einem Stromfluss innerhalb einer Faser (Wirbelströme) und zu Ringströmen in einem Fasernetzwerk (verbundene benachbarte Fasern) führt.

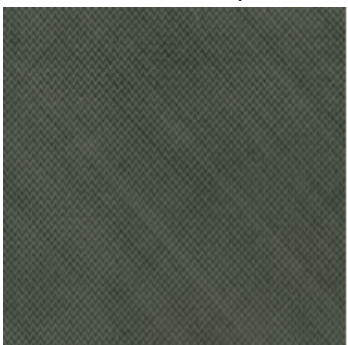

a)

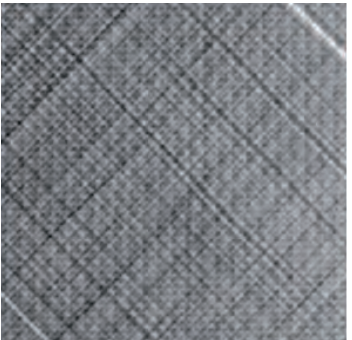

b)

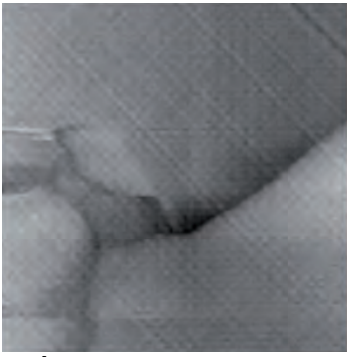

C)

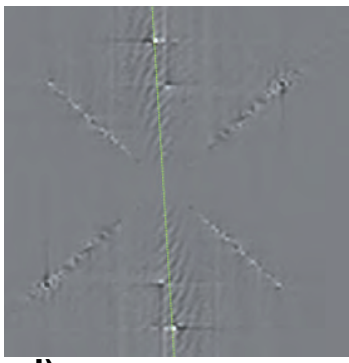

d)

Abb. 6 Photographie eines $30 \times 30 \mathrm{~cm}$ großen 5-Axial CF-Geleges, b) Realteil und c) Imaginärteil des Wirbelstromsignals der gleichen Probe. Bild d) zeigt eine 2d FFT des Realteils zur Texturanalyse.

Durch das Kalibrieren von sensorangepassten Parametersets für entsprechende CFK-Platten lassen sich bestimmte Schichten / Gelege ein- und ausblenden bzw. separieren. Die folgenden Abbildungen (Abb. 5) zeigen typische Defekte. Derzeit befindet sich ein multiparametrierbares Wirbelstromarray für die industrielle Anwendung in der Erprobung [6].
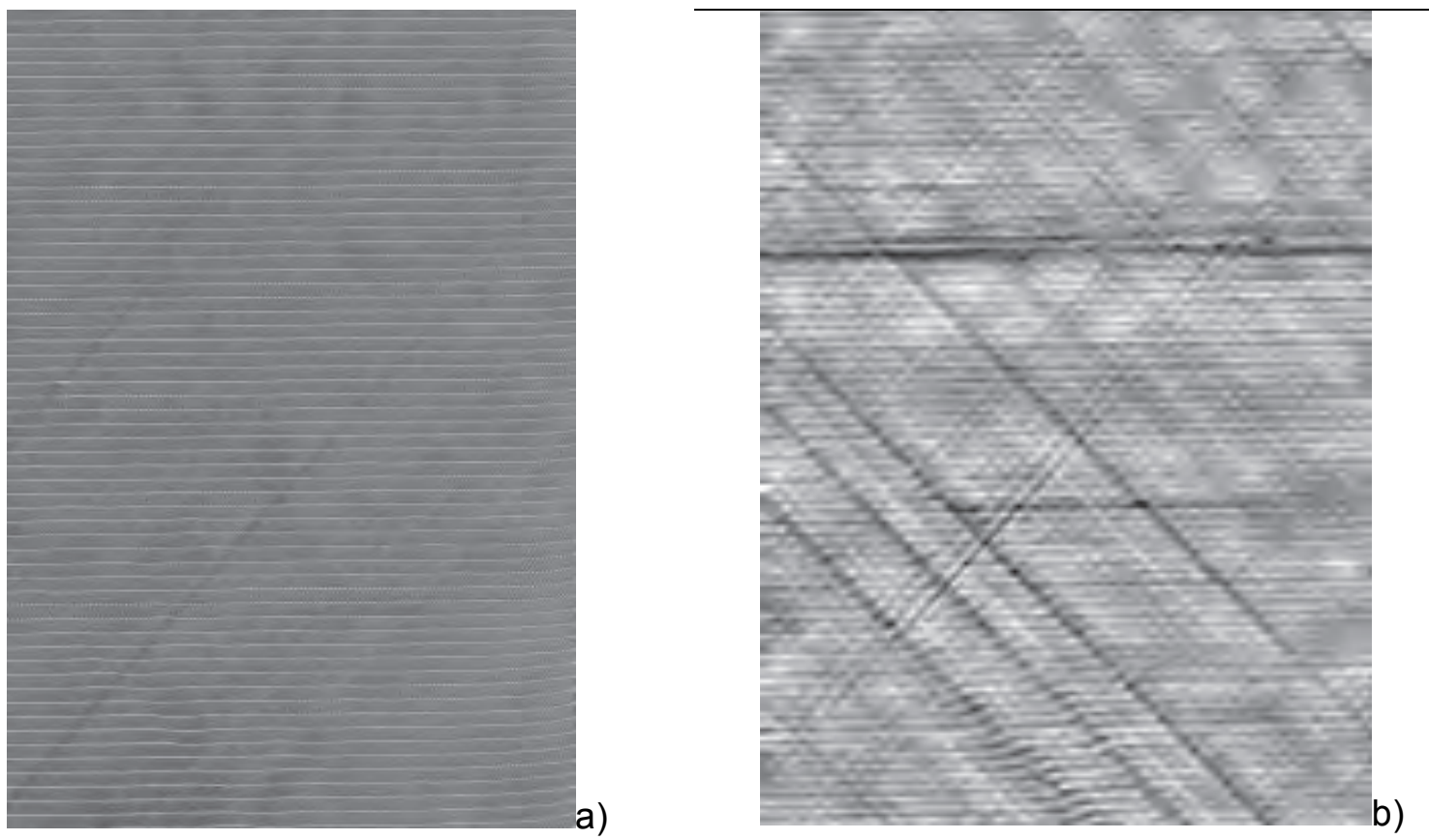

Abb. 7 Prüfung von CFK-Gelegen, 3-lagiges RCF mit Gasse in der 1. Lage, ein fehlender Querroving in der 2. Lage sowie mehrerer fehlender Rovings in der 3. Lage a) Foto von der Vorderseite, b) Hochauflösender Wirbelstromscan (Vorderseite), Auflösung ( 1x1mm)

\section{Dünnschichten und komplexe Dünnschichtsysteme}

Dünne Funktionsschichten werden in einer Vielzahl von Anwendungen wie beispielsweise der Elektronik, Dispnten Industrien sind in der Regel komplexe Mehrlagenaufbauten aus einer Vielzahl 
von dünnen Funktionsschichten, deren elektrische Eigenschaften und Defektfreiheit produktionsnah geprüft werden müssen.

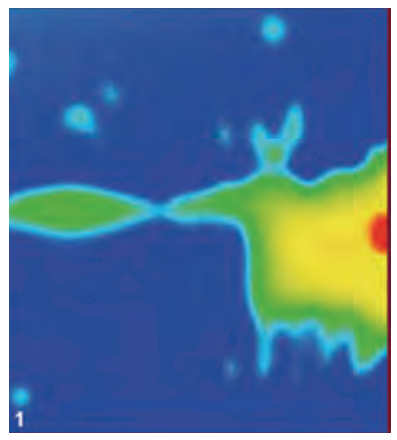

\title{
Abb. 8 Abbildung des Schichtwiderstandes einer TCO-Schicht (Transparent Conductive Oxide) auf Glas (10 x $10 \mathrm{~cm}, \sim 250 \mu \mathrm{m}$ Auflösung)
}

Ein wichtiger Qualitätsparameter von Dünnenschichtsystemen ist der elektrische Schichtwiderstand, der Einflüssen von z. B. Schichtdicke, Mikrostruktur, chemischer Zusammensetzung unterliegt. Sinnvoll ist ein Schichtwiderstandsmonlay- oder Photovoltaikindustrie eingesetzt. Durch die Notwendigkeit zur Kostenreduzierung bei gleichbleibend höchsten Anforderungen an Qualität und Zuverlässigkeit entstehen neue Anforderungen an die Prüftechnik zur Prozesssteuerung und Qualitätssicherung. Produkte aus den genanitoring insbesondere nach Abscheidungs- und Strukturierungsprozessen oder z. B. als finale Qualitätskontrolle. Ein leistungsfähiger Ansatz, um Informationen über den Schichtwiderstand und andere elektrisch charakterisierbare Größen zu erhalten, ist die hier vorgestellte hochauflösende abbildende Wirbelstromprüfung. Mit den Vorzügen der Wirbelstromtechnik (berührungslos, kontaktmittelfrei, schnell und geringer apparativer Aufwand) ist diese Methode gut in Produktionsprozesse integrierbar und arbeitet zerstörungsfrei.

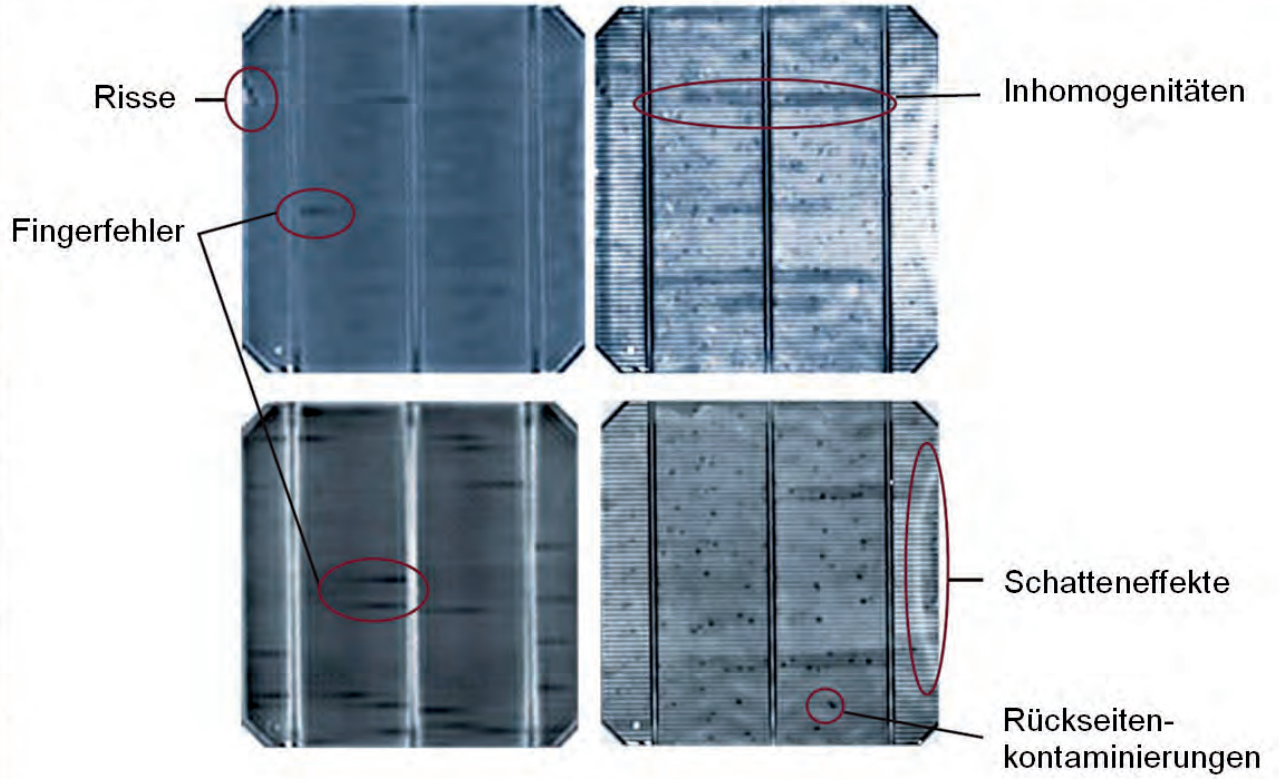

\begin{abstract}
Abb. 9 Hochauflösende abbildende Wirbelstromprüfung an monokristallinen SiliziumSolarzellen zeigen Fehler in der Elektrodenstruktur, Inhomogenitäten sowie Rückseitenverunreinigungen
\end{abstract}

Es wurde ein Gerät für den Einsatz im Labor entwickelt, mit dem Schichtdicken-schwankungen (Abb. 8), defekte Kontakte, Delaminationen, Kurzschlüsse, Risse, Fingerfehler, Verunreinigungen und Inhomogenitäten (Abb. 9 und Abb. 10) in dünnschicht- und waferbasierten Solarzellen nachweisbar sind [7]. 


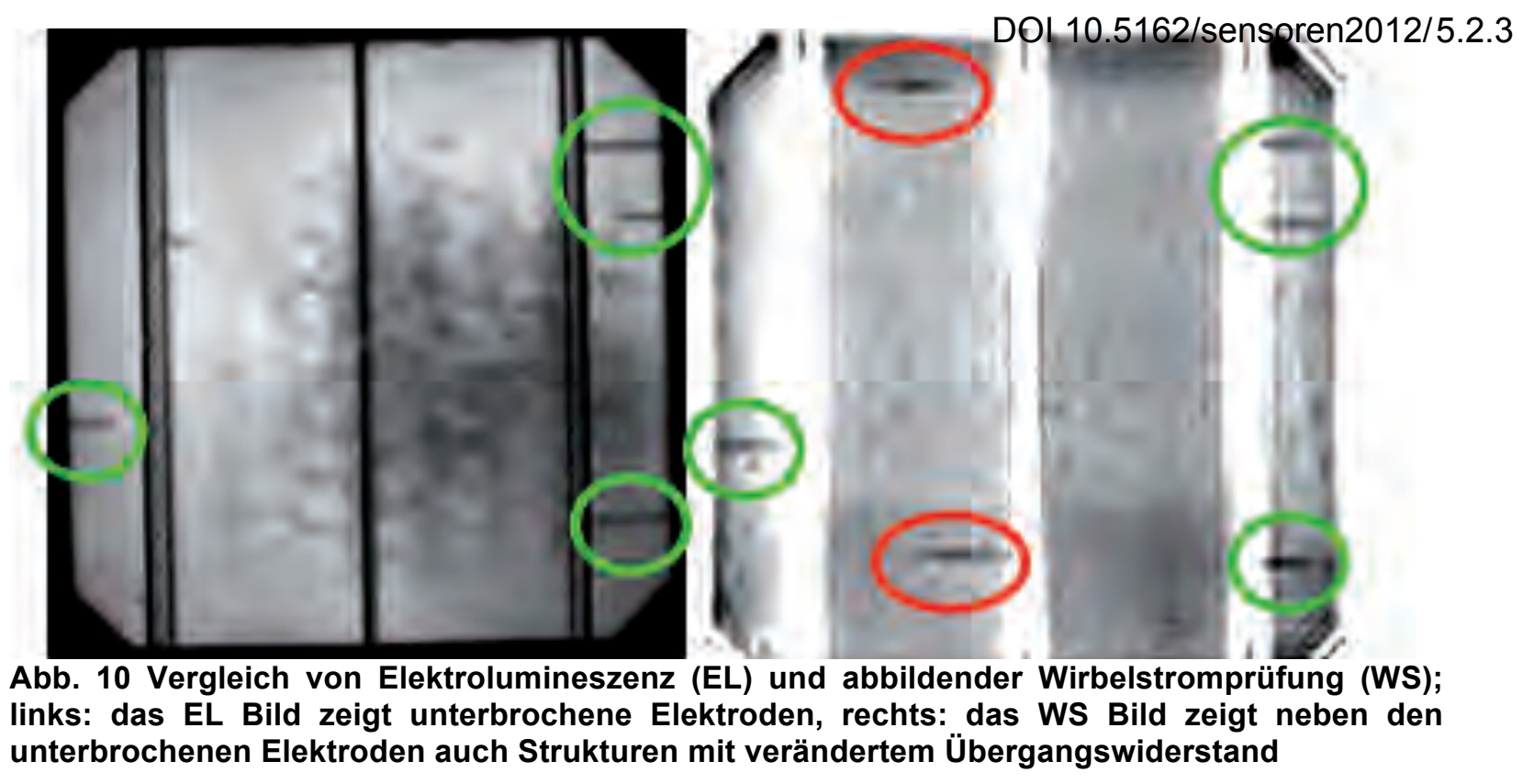

\section{Referenzen}

[1] H. Heuer, S. Hillmann, M. Röllig, M. H. Schulze, K-J. Wolter, „Thin Film Characterization Using High Frequency Eddy Current Spectroscopy", Conference on Nanotechnology, Genua, 2009

[2] A. Yashan, „Numerische Modellierung von Wirbelstromaufgaben und Lösung des inversen Problems“, ZfP-Zeitung 67, Oktober 1999

[3] A. Yashan, „Über die Wirbelstromprüfung und magnetische Streuflussprüfung mittels GMRSensoren“, Dissertation Universität Saarbrücken, 2008

[4] DIN 54140 Zerstörungsfreie Prüfung; Induktive Verfahren (Wirbelstromverfahren); Teil 1 04.76: Allgemeines; Teil 2 03.84: Begriffe: Teil 3 09.87: Darstellung und allgemeine Eigenschaften von Spulensystemen

[5] S. Hillmann, H. Heuer, N. Meyendorf, „High frequency eddy current device for near surface material characterizations“, Proc. of SPIE, Vol. 7293 729308-1, 2009

[6] M. H. Schulze, H. Heuer, M. Küttner, N. Meyendorf, "Mehrfrequenz-Wirbelstromprüfverfahren zur Qualitätskontrolle bei der Produktion von Kohlefaser-Multiaxialgelegen", Konferenzbeitrag DGZfPJahrestagung, 2009

[7] S. Hillmann, M. Klein, H. Heuer, „Schichtdicken-Charakterisierung dünner, leitfähiger Schichtsysteme mittels Wirbelstromtechnik“, DGZFP-Jahrestagung - Poster, 2010 